\title{
INVESTIGAÇÃO DA NOÇÃO DE CONSERVAÇÃO DE QUANTIDADES DISCRETAS EM PRÉ-ESCOLARES NASCIDOS PREMATUROS E A TERMO POR MEIO DO JOGO DE DOMINÓ
}

\author{
INVESTIGATION OF THE NOTION OF CONSERVATION OF \\ DISCREET QUANTITIES BETWEEN PREMATURE AND FULL \\ TERM PRE-SCHOOL CHILDREN BY MEANS OF THE GAME OF \\ DOMINO
}

\author{
Daiana Stursa $^{1}$ \\ Sávio Silveira de Queiroz ${ }^{2}$ \\ Sônia Regina Fiorim Enumo ${ }^{3}$
}

Stursa, D; Queiroz, SS.; Enumo, SRF. Investigação da noção de conservação de quantidades discretas em pré-escolares nascidos prematuros e a termo. Rev Bras Cresc e Desenv Hum 2010; 20(2): 238-249.

\section{Resumo:}

Considera-se prematuridade e baixo peso como elementos de risco ao desenvolvimento em seus aspectos neurológico, cognitivo, sensorial e motor infantil. A literatura aponta a Matemática como a área acadêmica mais prejudicada para o recém-nascido prematuro e com baixo peso em idade pré-escolar. Esta pesquisa investigou se crianças na faixa etária de cinco a cinco anos e 11 meses, nascidas prematuras e com baixo peso, apresentam a noção de conservação de quantidades discretas. Para isso, avaliou-se 24 crianças; 12 nascidas prematuras e com baixo peso (G1 - PT-BP) e 12 crianças nascidas a termo e com peso acima de 2.500 gramas (G2 - AT), por meio da Prova Operatória Piagetiana de Correspondência termo a termo e do jogo de Dominó. O procedimento de coleta de dados foi realizado em quatro sessões filmadas - uma para aplicação da Prova Operatória piagetiana e três para partidas de Dominó. Durante a coleta efetuou-se perguntas baseadas no Método Clínico piagetiano. Os dados foram tratados por análises descritivas e estatísticas. Os resultados apontaram desempenhos semelhantes entre os participantes de G1 (PT-BP) e G2 (AT) nos dois instrumentos utilizados, e que a noção de conservação de quantidades discretas não está plenamente construída, em relação ao esperado para a idade, segundo parâmetros da Epistemologia Genética.

Palavras-chave: Prematuridade; baixo peso ao nascimento; noção de conservação; jogo de dominó; epistemologia genética.

1 Mestre em Psicologia. Doutoranda do Programa de Pós-graduação em Psicologia, Universidade Federal do Espírito Santo. Email: daianastursa@yahoo.com.br

2 Professor Associado do Programa de Pós-graduação em Psicologia, Universidade Federal do Espírito Santo. E-mail: savioqueiroz@terra.com.br

3 Professora Associada do Programa de Pós-graduação em Psicologia, Universidade Federal do Espírito Santo. E-mail: soniaenumo@terra.com.br

Correspondência para: Daiana Stursa - Rua Oscar Rodrigues de Oliveira, $n^{0} 575$, apartamento 201, Edifício Premier, Jardim da Penha, Vitória-ES. CEP: 29.060-720. Telefone: (27) 3324-4869; (27) 9996-5930. Fax: (27) 4009-2501. Endereço eletrônico: daianastursa@yahoo.com.br. Mestrado com bolsa CAPES, Participação em Pesquisa Institucional CNPq - Processo 485564/ 2006-8. Artigo derivado de dissertação de Mestrado: “Avaliação da conservação de quantidades discretas em pré-escolares prematuros e a termo: um estudo investigativo com o jogo de Dominó”, apresentada em Agosto de 2008 ao Programa de Pósgraduação em Psicologia da Universidade Federal do Espírito Santo (PPGP-UFES). 


\begin{abstract}
:
Considered of great risk to a child's development, prematurity and low birth weight are elements that may affect neurological, cognitive, sensorial and motor development children. Literature points Mathematics as the most damaged academic area to the premature and low weight newborn in preschool age. This research has investigated if children in the age group of 5 to 5 years and 11 months old, that were born premature and with low weight, present the notion of conservation of discreet quantities. For that, 24 children were analyzed; 12 premature and with low birth weight (G1 - PT-BP) and 12 born to term and with birth weight above 2,500g (G2 - AT), through the Piagetian Operative Test of Correspondence one to one and the game Domino. The procedure of data collection was accomplished in four filmed sessions - one for the application of Piagetian Operative Test and three for the Domino matches. During the data collection, questions based on the Piagetian Clinical Method were asked. Descriptive and statistical analyses were used to deal with the data. The results point to a similar development between the participants of G1 (PT-BT) and G2 (AT) in both instruments used, and that the notion of conservation of discreet quantities is not fully constructed, concerning what was expected for the age, according to the parameters of Genetic Epistemology.
\end{abstract}

Key words: Prematurity; low birth weight; notion of conservation; game domino; genetic epistemology.

\section{INTRODUÇÃO}

Os conceitos de prematuridade e de baixo peso atualmente adotados pela Organização Mundial de Saúde (OMS) são, respectivamente, a condição do bebê nascido até 36 semanas e 6 dias e nascimento com peso inferior a 2.500 gramas $^{1}$.

Sendo o baixo peso ao nascer o melhor indicador da saúde neonatal e do desenvolvimento ${ }^{2}$ do recém-nascido, torna-se, portanto, imprescindível introduzir o mais rapidamente possível a alimentação enteral e parenteral, para não que não se modifique, negativamente, o estado nutricional, minimizando perdas e estimulando a maturação do trato gastrointestinal. Também é importante o follow-up relativo ao crescimento e desenvolvimento do recém-nascido prematuro e baixo-peso para que não haja prejuízo nutricional ${ }^{3}$.

Dados do Ministério da Saúde revelam aumento no índice de nascimento pré-termo nos últimos anos. Dos 3.035.094 nascidos vivos em 2005, 199.018 foram prematuros, enquanto em 2002 esse número foi de 192.566 para o total de 3.059.402 crianças nascidas, resultando ainda em 34.213 nascimentos com peso abaixo de 1.500 gramas, o que equivale a $17 \%$ do número de crianças prematuras ${ }^{4}$.
A prematuridade associada ao baixo peso no nascimento tende a ser considerada como importante demanda de saúde coletiva, em razão da sua prevalência em torno de $10 \%$ da população, em quase todo o mundo, independentemente das diferenças de níveis de desenvolvimento entre os países ${ }^{5,6}$.

Além disso, a associação entre peso e idade gestacional no nascimento dos recémnascidos prematuros e baixo-peso é indispensável para a previsão de problemas que levam à mortalidade, morbidade ou dificuldades no desenvolvimento, uma vez que tais fatores desempenham importante papel na maturação de vários sistemas do organismo. ${ }^{7}$ Assinala-se que, quanto mais baixo o peso de nascimento, maior a possibilidade de ocorrerem distúrbios, déficits e problemas no desenvolvimento neurológico, cognitivo, sensorial e motor na criança ${ }^{8-15}$.

As deficiências emocionais, comportamentais, cognitivas e fisiológicas estão associadas, durante a infância, respectivamente, aos seguintes prejuízos: timidez e ansiedade; hiperatividade, baixa autoconfiança e menor vínculo social; dificuldades 
de aprendizagem, fraca concentração, alteração na memória, problemas de comportamento na escola e baixo escore em testes de QI; baixa estatura, obesidade e doenças metabólicas; e problemas no crescimento ${ }^{6,11-13,16-21}$. Com referência ao desempenho acadêmico, a Matemática, mais especificamente a Aritmética, mostrou-se a área mais prejudicada, à medida que o peso ao nascer diminui ${ }^{13-14,22-23}$.

A educação matemática, com referencial construtivista, parte da proposição de que o conhecimento lógico-matemático é inventado por cada criança na interação com o meio e nas descobertas arroladas na experiência ati$\mathrm{va}^{24-26}$. Assim, na formação do conceito de número estão envolvidos os conhecimentos físicos e lógico-matemáticos por meio da abstração reflexiva ${ }^{27}$.

O conceito de número encontra-se na origem de todo conhecimento matemático que será desenvolvido no ensino escolarizado. Portanto, é importante assegurar sua construção pela criança em idade pré-escolar, uma vez que a Matemática é apontada como a disciplina com o maior índice de reprovação para todos os estudantes, principalmente, no ensino fundamental ${ }^{28}$.

Para que haja compreensão de qualquer número, a criança precisa estabelecer a relação quantitativa entre os elementos e o número que representa essa quantidade ${ }^{29}$. Obedecendo aos processos construtivos das noções e características do estádio pré-operacional ${ }^{27}$, desde os primeiros anos de sua vida até, aproximadamente, os sete anos de idade, são necessárias quatro condições para formação do conceito de número pela criança: conservação de quantidades; a correspondência termo a termo, essencial para a contagem; determinação do valor cardinal; e determinação do princípio ordinal ${ }^{29}$. A conservação ${ }^{1}$ de quantidades, por sua vez, é basal para engendrar o conceito de número, já que só se compreende um número desde que ele permaneça idêntico a si mesmo, seja qual for a disposição espacial assumida por suas unidades ${ }^{29}$.
A hipótese inicial de pesquisa é a de que crianças nascidas prematuras e com baixo peso tendem a apresentar defasagem ao construírem a noção de conservação de quantidades discretas, se comparadas a crianças nascidas a termo, que não apresentaram problemas no nascimento, seguindo indicações da literatura que aponta problemas na área de Matemática para crianças com condições adversas ao nascimento $^{20,22-23}$.

Assim, o objetivo é verificar se crianças nascidas prematuras e com baixo peso, em idade pré-escolar, apresentam noção de conservação de quantidades discretas e se prematuridade e o baixo peso ao nascer estão relacionados com dificuldades na construção da noção de conservação de quantidades discretas e formação do conceito de número.

\section{MÉTODO}

Para responder aos objetivos, nesta pesquisa avaliou-se e comparou-se a noção de conservação de quantidades discretas de 24 préescolares $^{2}$ com idade entre 5 anos e 5 anos e 11 meses divididos em dois grupos. O primeiro grupo (G1) foi composto por sete meninos e cinco meninas nascidos prematuros (idade gestacional de 28 a 36 semanas); com baixo peso (peso entre 900 a 2.355 gramas) e que tiveram internação em UTIN ${ }^{3}$.

O segundo grupo (G2) foi composto por sete meninos e cinco meninas nascidos a termo (idade gestacional de 37 a 40 semanas); com peso normal (peso entre 2.690 a 4.210 gramas); e que não apresentaram problemas no nascimento. Todos os participantes de G1 eram prematuros e baixo-peso (PT-BP), e participavam do Programa de Follow-up neurológico de um hospital localizado em um município da região da Grande Vitória/ES e vinculado ao Sistema Único de Saúde (SUS). Todos os participantes de G2 eram crianças nascidas a termo (AT), ou seja, nascidas com idade 
gestacional igual ou superior a 37 semanas e estavam matriculadas em uma pré-escola ${ }^{4}$ localizada no mesmo município do hospital citado.

A constituição de ambas as amostras foi por conveniência ${ }^{5}$ e com a cooperação e autorização dos pais ou responsáveis legais.

Os instrumentos utilizados foram a Prova Operatória Piagetiana de Correspondência Termo a Termo ${ }^{29}$ e uma modalidade do jogo de Dominó jogado em três partidas. A coleta foi realizada em quatro sessões filmadas. A Prova Operatória Piagetiana utilizada objetiva avaliar a conservação de quantidades discretas à base do grau de conservação de dois conjuntos de fichas (de cores diferentes, mas com mesma quantidade de elementos), independentemente da disposição espacial que eles assumam.

De acordo com as respostas obtidas, o participante é classificado em três níveis de operacionalidade: conservador (escore 8 a 6); intermediário (escore 5 a 3); e não conservador (escore 2 a zero) $)^{30}$.

A modalidade de jogo de Dominó utilizada foi desenvolvida especificamente para esta pesquisa, com peças variando de 2 a 8 unidades e cujos sulcos que as representam possuem configurações diferentes para uma mesma quantidade. O jogo de Dominó foi avaliado considerando-se a possibilidade de ocorrência de dez tipos de erros diferentes, com tipificação fundamentada nos níveis de compreensão de jogo estabelecido por Jean Piaget ${ }^{31}$.

A descrição dos tipos de erros está detalhada na Quadro 1. O jogo de Dominó também foi avaliado considerando os níveis de compreensão de jogo ${ }^{32}$ os quais estão descritos na Quadro 2.

A coleta de dados e parte da análise foi baseada no Método Clínico piagetiano, pelo qual utiliza-se sistematização das intervenções nos procedimentos baseadas em observações sobre as ações do sujeito, por meio de perguntas de exploração, justificação e controle. ${ }^{33-36}$ Tais intervenções permitem tanto a construção
Quadro 1: Tipos de erro no jogo de Dominó utilizado

\begin{tabular}{|cl|}
\hline $\begin{array}{c}\text { Tipo } \\
\text { de erro }\end{array}$ & \multicolumn{1}{c|}{ Descrição } \\
\hline E1 & $\begin{array}{l}\text { Não conseguir contar a quantidade da peça } \\
\text { com os dedos. }\end{array}$ \\
\hline E2 & $\begin{array}{l}\text { Jogar peça sem relação com o valor das peças } \\
\text { da extremidade e, conseqüentemente, com a } \\
\text { jogada atual. }\end{array}$ \\
\hline E3 & $\begin{array}{l}\text { Encaixar, propositalmente, peça de valor } \\
\text { diferente da requisitada pela jogada atual para } \\
\text { não precisar comprar nova peça. }\end{array}$ \\
\hline E4 & $\begin{array}{l}\text { Contar as próprias peças a procura de uma } \\
\text { para jogar, mas sem prestar atenção na } \\
\text { quantidade da peça de cada extremidade do jogo. } \\
\text { Contar somente um lado da peça para verificar }\end{array}$ \\
\hline E5 & $\begin{array}{l}\text { se esta possui ou não a quantidade para ser } \\
\text { jogada no Dominó, ignorando a outra } \\
\text { extremidade da própria peça. }\end{array}$ \\
\hline E6 & $\begin{array}{l}\text { Comprar peça desnecessária, pois já possui } \\
\text { uma para efetuar jogada. } \\
\text { Não olhar todas as peças que possui ao fazer }\end{array}$ \\
\hline E7 & $\begin{array}{l}\text { uma jogada (a fim de escolher aquela que mais } \\
\text { convém). }\end{array}$ \\
\hline E8 & $\begin{array}{l}\text { Não conseguir perceber mesma quantidade em } \\
\text { peças com diferente disposição dos sulcos. }\end{array}$ \\
\hline E9 & $\begin{array}{l}\text { Mostrar peça ao adversário por distração ou } \\
\text { propositalmente. }\end{array}$ \\
\hline E10 & $\begin{array}{l}\text { Encaixar peça de valor diferente da } \\
\text { extremidade do Dominó com o intuito de ficar } \\
\text { sem peças e ganhar o jogo. }\end{array}$ \\
\hline
\end{tabular}

Quadro 2: Níveis de compreensão do jogo de Dominó

\begin{tabular}{|c|c|}
\hline Nível & Descrição \\
\hline I & $\begin{array}{l}\text { Não jogar de acordo com as regras do jogo; } \\
\text { Não conseguir contar sozinho a quantidade } \\
\text { expressa na peça; Não reconhecer que uma } \\
\text { quantidade "X” pode estar representada de uma } \\
\text { forma "A” em uma peça e de uma forma "B” } \\
\text { em outra peça e, que no jogo, essas peças } \\
\text { podem ser encaixadas uma na outra; Mostrar } \\
\text { as peças para a experimentadora pedindo ajuda } \\
\text { para efetuar uma jogada correta; Se fixar em } \\
\text { um dos lados do jogo em sucessivas jogadas. }\end{array}$ \\
\hline II & $\begin{array}{l}\text { Conseguir contar a quantidade expressa na } \\
\text { peça com o auxílio dos dedos; Reconhecer que } \\
\text { uma quantidade “X” pode estar representa de } \\
\text { uma forma “A” em uma peça e de uma forma } \\
\text { "B”, em outra peça, ao efetuar a contagem } \\
\text { dessas peças; Não olhar os dois lados do jogo } \\
\text { para escolher em qual irá efetuar a jogada; } \\
\text { Não reconhecer que sua peça pode ser jogada } \\
\text { nos dois lados do jogo; Não reconhecer que é } \\
\text { melhor jogar a peça com ‘bomba’ em qualquer } \\
\text { que seja a jogada. }\end{array}$ \\
\hline III & $\begin{array}{l}\text { Conseguir contar mentalmente a quantidade } \\
\text { expressa na peça; Reconhecer que uma } \\
\text { quantidade “X” pode estar representa de uma } \\
\text { forma "A" em uma peça e de uma forma "B” } \\
\text { em outra peça e, mesmo assim, possuírem } \\
\text { mesma quantidade; Considerar os dois lados } \\
\text { do jogo ao efetuar uma jogada; Procurar entre } \\
\text { suas peças a melhor para efetuar uma jogada. }\end{array}$ \\
\hline
\end{tabular}


como a verificação de hipóteses pelo experimentador, em processo dialético constante, entre as lógicas do entrevistador e do sujeito $^{36}$.

De modo detalhado, as perguntas de exploração (PE) investigam o domínio das regras, estratégias e informações do jogo. Suas respostas são divididas em domínio presente (DP) e domínio ausente (DA). Na primeira, a criança possui entendimento das regras, excluindo impossibilidades e antecipando erros; na segunda, não existe compreensão nem das regras e nem das possibilidades.

As perguntas de justificação (PJ), por sua vez, verificam a qualidade de reflexão e argumentação das ações e investigam se a jogada realizada insere-se dentro de um programa de jogo. As respostas podem ser de legitimação argumentada (LA), fundamentadas por uma boa explicação e, de legitimação não-argumentada (LNA), sem boa fundamentação.

As perguntas de controle exploram o grau de estabilidade das aquisições do entrevistado. O intuito, portanto, é observar se o sujeito mantém sua jogada e seu esquema de jogo ou se os modifica quando questionado quanto à sua jogada. As respostas são divididas em: coerência forte $(\mathrm{C}+)$, em que a pergunta não causa desequilíbrio ou contradição, e em coerência fraca (C-), em que há presença de contradição e pseudo-necessidades na criança.

As respostas de coerência fraca possibilitam nova pergunta de controle, que se vale a observar a contra-argumentação do entrevistado, podendo gerar respostas de contradição forte $(\mathrm{T}+)$, em que as resistências são ultrapassadas e uma melhor estratégia é incorporada; e em contradição fraca (T-), em que o sujeito não se afeta pela pergunta nem modifica sua conduta e ponto de vista ${ }^{33,36}$.

Os dados também foram tratados por análise estatística que, pelo tamanho reduzido da amostra, exigiu adoção de testes nãoparamétricos. Todas as análises foram realizadas inserindo os resultados (escore e classificação) em planilhas do programa SPSS ${ }^{\circledR 37}$. Os testes nãoparamétricos usados foram: a) Binomial, b) Mann-Whitney e c) qui-quadrado.

\section{RESULTADOS}

Os resultados obtidos na Prova Operatória Piagetiana de Correspondência Termo a Termo dos participantes de G1 (PT-BP) mostraram que 11 deles apresentaram nível operatório não conservativo e um apresentou nível intermediário (escore 5). Quatro participantes de G2 (AT) apresentaram nível operatório intermediário (escore 3 a 5) e oito nível não conservativo. O Teste de Mann-Whitney não detectou diferença estatisticamente significativa $(p=0,322)$ entre as médias dos dois grupos nesse instrumento.

A divisão das partidas do jogo de Dominó em jogadas permitiu estabelecer os seguintes parâmetros: os 12 participantes de G1 (PT-BP) efetuaram um total de 344 jogadas nas 36 partidas, com média de 9,56 jogadas por partida (valores: mínimos de seis e máximo de 15 jogadas). Enquanto os 12 participantes de G2 (AT) efetuaram total de 315 jogadas nas 36 partidas, com média de 8,75 jogadas por partida (valores: mínimo de 5 e máximo de 13 jogadas).

A categorização das perguntas realizadas a G1 (PT-BP) mostrou que das 561 perguntas realizadas, 364 referiam-se às perguntas de exploração (PE), 122 às de justificação (PJ) e 75 às de controle (PC). Enquanto que das 637 perguntas realizadas a G2 (AT), 391 foram classificadas como PE, 169 como PJ e às 77 como PC. O Método Clínico aplicado com base na teoria piagetiana, naturalmente conduz o experimentador em postura dialética que implica na quase impossibilidade de padronização do número de perguntas para ambos os grupos, o que justifica as diferenças 
nas quantidades de perguntas efetuadas aos participantes de ambos os grupos.

Não houve diferença estatisticamente significativa, usando o Teste de MannWhitney, na comparação das respostas de domínio, legitimação e controle entre G1 (PTBP) e G2 (AT), para cada partida do jogo de Dominó. Também não houve diferença estatisticamente significativa pelo Teste Binomial entre os dois grupos, comparandose as proporções das respostas apresentadas pelos participantes às perguntas de exploração, justificação e controle na totalização das três partidas de Dominó. Os níveis de significância encontrados foram, respectivamente, $\mathrm{p}$-valor $=0,180472$; $\mathrm{p}$-valor $=$ 0,109295 e p-valor $=0,442626$ para as categorias de domínio, legitimação e coerência.

A categorização dos erros durantes as três partidas revelou que os participantes de G1 (PT-BP) apresentaram total de 730 erros enquanto G2 (AT) total de 671. Os valores di-

Tabela 1: Erros cometidos nas três partidas de Dominó pelos participantes de G1 (PT-BP) e G2 (AT)

\begin{tabular}{|c|c|c|c|c|c|c|c|c|c|}
\hline \multirow[t]{2}{*}{$\begin{array}{l}\text { Tipo de } \\
\text { erro }\end{array}$} & \multicolumn{2}{|c|}{ Partida inicial } & \multicolumn{2}{|c|}{$\begin{array}{c}\text { Partida } \\
\text { intermediária }\end{array}$} & \multicolumn{2}{|c|}{$\begin{array}{c}\text { Partida } \\
\text { final }\end{array}$} & \multicolumn{2}{|c|}{$\begin{array}{l}\text { Soma das } \\
\text { três partidas }\end{array}$} & \multirow[t]{2}{*}{ Total } \\
\hline & G1 & G2 & G1 & G2 & G1 & G2 & G1 & G2 & \\
\hline E1 & 15 & 21 & 12 & 18 & 16 & 17 & 45 & 63 & 108 \\
\hline E2 & 34 & 24 & 33 & 24 & 28 & 21 & 95 & 71 & 164 \\
\hline E3 & 7 & 0 & 6 & 3 & 3 & 4 & 16 & 7 & 23 \\
\hline E4 & 33 & 21 & 37 & 32 & 38 & 31 & 108 & 86 & 192 \\
\hline E5 & 18 & 6 & 19 & 14 & 21 & 11 & 58 & 31 & 89 \\
\hline E6 & 6 & 6 & 2 & 4 & 9 & 9 & 17 & 17 & 36 \\
\hline E7 & 70 & 87 & 71 & 85 & 73 & 74 & 214 & 248 & 460 \\
\hline E8 & 27 & 33 & 29 & 31 & 25 & 28 & 81 & 95 & 173 \\
\hline E9 & 18 & 10 & 23 & 28 & 18 & 9 & 59 & 47 & 106 \\
\hline E10 & 10 & 0 & 14 & 6 & 13 & 7 & 37 & 13 & 50 \\
\hline Total & 238 & 208 & 246 & 245 & 244 & 211 & 730 & 678 & 1401 \\
\hline
\end{tabular}

vididos por tipo de erro estão detalhados na Tabela 1.

O Teste de Mann-Whitney foi usado de duas maneiras diferentes na comparação dos tipos de erro apresentados nas partidas de Dominó pelos dois grupos. Cada uma das três partidas foi avaliada em separado, mas, também, o total dos valores nelas apresentados foi reunido para se verificar se os resultados encontrados nas análises em separado apresentavam alguma diferença estatisticamente significativa. Na partida inicial houve diferença estatisticamente significativa para o erro E7 $(\mathrm{p}=0,010)$ cometido pelos participantes de G2 (AT). Na partida intermediária houve diferença estatisticamente significativa novamen- te para o erro E7 $(p=0,010)$ em relação aos participantes de G2 (AT). Na partida final o teste apontou diferença estatisticamente significativa para o erro E5 $(\mathrm{p}=0,078)$ cometido pelos participantes de G1 (PT-BP).

A confrontação dos erros reunidos das três partidas de Dominó entre dois grupos determinou diferenças estatisticamente significativas para os erros E5 ( $p=0,016)$, E7 (p-valor= 0,000) e E10 ( $p=0,003)$, uma vez que os participantes de G1 (PT-BP) erraram mais ao deixarem de contar ambos os lados de suas peças, ao procurar uma delas para efetuar jogada, do que os participantes de G2 (AT). Quanto ao alto índice de ocorrência do erro E7, relaciona-se ao fato de que os participantes de G2 (AT), ao encon- 
trarem peça compatível à extremidade do Dominó, logo efetuavam jogada, deixando de olhar e procurar se, dentre suas outras peças, havia uma opção melhor para realizar tal joga$\mathrm{da}$, conduta adotada mais vezes pelos participantes de G2 (AT) do que pelos de G1 (PT-BP). $O$ resultado encontrado para o erro E10 sofreu influência pelas jogadas de alguns participantes de G1 (PT-BP) que o cometeram sistematicamente. Na tentativa de ganhar a partida, esses se desfaziam de suas peças sem a observância das regras do jogo de Dominó mais vezes que os participantes de G2 (AT).

Analisando e classificando os níveis de compreensão das partidas do jogo de Dominó dos participantes de G1 (PT-BP), encontrouse os seguintes resultados: a maioria dos participantes (83,3\%) apresentou, em pelo menos uma das partidas, nível II de compreensão de jogo, sendo predominante para seis participantes (50\%). Os resultados do nível de compreensão de jogo dos participantes de G2 (AT) revelaram que todos apresentaram, em pelo menos uma partida, o nível II de compreensão. Esse mesmo nível foi ainda dominante para sete participantes desse grupo (58,3\%). Portanto, a maior parte dos participantes dos dois grupos conseguiu jogar contando as peças de Dominó com o auxílio dos dedos, não apresentaram visão geral da partida e nem conservação da quantidade expressa nas peças, como demonstra a Tabela 2. O Teste $\chi$-quadrado de Pearson, não detectou diferenças estatisticamente significativas nos níveis de compreensão do jogo de Dominó entre os dois grupos de crianças ava$\operatorname{liados}\left(\chi^{2}=2,67\right.$ e $\left.\mathrm{p}=0,264\right)$.

\section{DISCUSSÃO}

Pelos resultados obtidos a partir da Prova Operatória Piagetiana de Correspondência Termo a Termo demonstrou-se que os participantes dos dois grupos basearam seus julgamentos nas aparentes dimensões que as duas
Tabela 2: Níveis de compreensão de jogo nas três partidas de Dominó apresentados pelos participantes de G1 (PT-BP) e G2 (AT)

\begin{tabular}{|c|c|c|c|c|}
\hline \multirow{2}{*}{ Grupo } & \multirow{2}{*}{ Participante } & \multicolumn{3}{|c|}{ Partida } \\
\hline & & Inicial & Intermediária & Final \\
\hline \multirow{12}{*}{ G1 (PT-BP) } & Gus & I & I & I \\
\hline & Pam & II & II & II \\
\hline & Kat & II & II & III \\
\hline & Ped & III & II & II \\
\hline & Clau & I & I & I \\
\hline & Marc & II & II & II \\
\hline & Ric & I & II & I \\
\hline & Fern & II & II & II \\
\hline & Wash & II & II & II \\
\hline & Mar & II & II & II \\
\hline & Rod & II & II & II \\
\hline & Marj & I & II & I \\
\hline \multirow{12}{*}{ G2 (AT) } & Mir & $\bar{I}$ & II & II \\
\hline & Ing & II & II & II \\
\hline & Get & II & I & I \\
\hline & Fran & II & II & III \\
\hline & Sau & II & II & II \\
\hline & Iva & I & II & II \\
\hline & Mar & II & II & II \\
\hline & Leo & I & II & II \\
\hline & Mat & II & II & II \\
\hline & Afon & II & II & II \\
\hline & Ren & II & II & II \\
\hline & Pau & II & II & II \\
\hline
\end{tabular}

coleções de fichas assumiram ao longo do procedimento, não mantendo em todos os momentos as equivalências quantitativa e qualitativa dos conjuntos de fichas. Deve-se considerar que, dentre as quatro condições envolvidas na formação do conceito de número, a conservação de quantidades discretas deve ser alcançada primeiramente. As dificuldades daí decorrentes poderão aparecer no contexto normal da escolarização. Ou seja, os procedimentos de ensino poderão não levar em conta essa lacuna cognitiva e apresentar os demais conteúdos escolares como se a criança já dominasse os 
anteriores, o que, no caso dos nossos participantes, ainda estão longe de consolidar-se. Um autêntico "efeito dominó" referente a acúmulo de dificuldades de aprendizagem pode, então, deflagrar-se.

Retomando as análises efetuadas para as perguntas de exploração, justificação e controle, pode-se afirmar que geraram respostas que conduzem a resultados que não expressam diferenças estatisticamente significativas. Desse modo pode-se inferir que ambos os grupos demonstram dificuldades para proceder sob “domínio presente”, para legitimar suas respostas, para elaborar sob coerência, e que as contradições inseridas não surtem efeito para melhoria de suas ações.

Quanto aos resultados encontrados para os erros, exceto aqueles já descritos como apresentando diferenças estatisticamente significativas, pode-se inferir, a partir da observação qualitativa e até verificando resultados gerais ou específicos tabulados (independentemente do tratamento estatístico efetuado) que os participantes de ambos os grupos não apresentaram melhoras em seus procedimentos.

Um fator que deve ser considerado como possível explicação a respeito de não terem sido encontradas diferenças estatisticamente significativas entre os dois grupos avaliados, relaciona-se aos avanços científicos e tecnológicos aplicados aos cuidados de crianças nascidas prematuras e baixo peso. Dentre esses, destacam-se as estimulações sensório-motoras realizadas pelas equipes multidisciplinares e o aperfeiçoamento da administração de alimentação enteral e parenteral, minimizando, paulatinamente, as dificuldades iniciais apresentadas pela condição de nascimento prematuro e com baixo peso.

Verifica-se ainda que os erros não se tornaram observáveis para as crianças e, tampouco, efeitos de aprendizagem a partir das ações sucessivas de jogar (treino) foram dignos de nota. Na partida inicial o erro E7 foi o mais cometido pelos participantes de G2 (AT), pois eles sistematicamente esqueciam de conferir todas as suas peças antes de efetuarem uma jogada, ao contrário dos participantes de G1 (PT-BP). Na partida intermediária novamente o erro E7 foi o mais cometido pelos participantes de G2 (AT) mostrando, mais uma vez, que eles efetuavam jogadas apressadamente, deixando de olhar e procurar se, entre suas outras peças, havia uma opção melhor para realizar a jogada.

E7 foi mais cometido pelos participantes de G2 (AT) nas duas primeiras partidas. Também foi o erro com maior índice de ocorrência para ambos os grupos, dentre todos os tipos de erros, e pode ser explicado pela ausência de visão global e de plano de jogo por parte dos participantes, uma vez que somente dois participantes de G1 (PT-BP) e um de G2 (AT) foram classificados com nível III (em uma partida dentre as três jogadas) de compreensão de jogo. Vale lembrar que, em muitas das vezes, este erro foi computado não porque o participante efetuava jogada errada, mas porque deixava de observar todas suas peças para escolher aquela com que faria a melhor jogada. Relacionamos isso com a agitação e a dificuldade de concentração que parcela considerável dos participantes de G1 (PT-BP) apresentou durante a coleta e ao desinteresse que parte dos participantes de G2 (AT) também apresentou durante a coleta.

Na partida final o erro E5 foi mais cometido pelos participantes de G1 (PT-BP) que deixaram de contar ambos os lados de suas peças ao procurar uma delas para efetuar uma jogada. Isso ocorreu mais vezes em G1 (PT$\mathrm{BP}$ ) do que para os participantes de G2 (AT). A abordagem utilizada nesta pesquisa permitiu inferir algo sobre o processo de construção desses conhecimentos, não apenas por observação escalar ou numérica, mas, sobretudo, pela observação de aspectos qualitativos sobre os recortes do desenvolvimento tais como foram considerados. Em geral, as crianças do grupo G1 (PT-BP) em comparação com as de 
G2 (AT), apresentaram comportamento agitado; desatenção; não cooperação com a experimentadora; dificuldade em lembrar regras e maior tendência a burlá-las. A agitação pode relacionar-se a um quadro de baixa tolerância à frustração por parte desses participantes, reflexo de educação mais tolerante e do excesso de proteção advindo dos pais e de Transtorno de Atenção e Hiperatividade. Desse modo, o resultado referente a E5 provavelmente está relacionado com tais comportamentos.

Como relatamos anteriormente nos resultados, os erros E5, E7 e E10 apresentaram diferenças estatisticamente significativas, pelo Teste de Mann-Whitney, na totalização das partidas. Entretanto, a justificativa para os resultados relativos aos erros E5 e E7 são os mesmos que os abordados nas partidas em separado. Assim, cabe apenas abordar o erro E10 a fim de se evitar redundâncias. O erro E10 foi mais cometido pelos participantes de G1 (PT$\mathrm{BP}$ ) do que pelos de G2 (AT), pois alguns participantes de G1 (PT-BP) se desfaziam de suas peças para tentar ganhar a partida, sem a observância das regras do jogo. Esses mesmos participantes demonstraram agitação e impaciência, principalmente nos minutos finais de cada partida. Quando eram questionados sobre suas jogadas, tinham o comportamento de: dar uma justificativa infundada; ficar calado e não justificar sua ação; dizer que era vez da experimentadora jogar; ou dizer que haviam ganhado a partida.

Com base na interpretação dos resultados concernentes aos erros apresentados no jogo e suas relações com os níveis de compreensão de jogo, constata-se que quase todos os participantes, de ambos os grupos (com exceção da participante Iva de G2 - AT), que cometeram muito freqüentemente os erros E1 e E2, foram classificados no Nível I de compreensão de jogo. Ao demonstrarem dificuldade em jogar o Dominó, não conseguindo contar as peças (seja mentalmente ou com os dedos) ou apreender que peças com formatos diferentes possuíam mesma quantidade, efetuavam jogadas arbitrárias. Constata-se que esses participantes tinham como estratégia exclusiva livrar-se de suas peças por meio da utilização de qualquer jogada, em detrimento ao respeito das regras.

Também, para a maioria desses participantes, os erros E4 e E5 (efetuar uma jogada sem considerar as condições básicas que uma jogada exige, ou seja, considerar os valores de sua peça e os valores de cada extremidade do jogo), embora menos primários que os erros E1 e E2, são insuficientes para promoverem ascensão para além do Nível I. Evidenciam-se na relação entre os erros E1, E2, E4, E5 e Nível I, aspectos de centração impeditivos do desenvolvimento cognitivo do sujeito. Inferese que esses participantes não possuíam boa compreensão de jogo, pois, saber contar suas peças e verificar a configuração das duas extremidades do jogo é fundamental para se realizar jogadas corretas, assim como realizar boas jogadas, além de assegurar bom desempenho na partida.

Os participantes com Nível II, de ambos os grupos, apresentaram desempenho mediano nas partidas de Dominó. Cometeram erros dos tipos E2, E4, E5, E6, E7, E8 e E9, com predomínio do erro tipo E7, mas com baixa freqüência (com máximo de ocorrência de 2 erros por partida, considerando cada tipo de erro). É importante informar que, a maior freqüência de erros de um mesmo tipo, foi registrada na partida inicial, mas, também, foi assistemática e decrescente, tendo ocorrido erros diferentes para os participantes de G1 (PT-BP) e G2 (AT). Esses participantes agrupados no Nível II não conseguem atingir a noção de conservação de quantidades discretas em decorrência da presença dos erros acima referidos, mas, principalmente, devido aos dos tipos E2 e E8. Conclui-se que eles apenas ascendem de nível por conseguir contar as peças e por não 
efetuarem, com grande freqüência, um mesmo tipo de erro. De qualquer modo, ainda muito distante de consolidar-se a noção plena de conservação de quantidades discretas.

Por observação qualitativa pode-se inferir também que, os participantes de G2 (AT) jogaram um pouco melhor que os de G1 (PT-BP) apenas porque souberam aproveitar os recursos advindos do "fator sorte", tão presente em jogos como o Dominó. Os participantes de G2 (AT) conseguiam identificar uma peça que servia ao encaixe porque contavam corretamente as quantidades e se convenciam rapidamente de sua validade. Contrariamente, os participantes de G1 (PT-BP), ou contavam as quantidades erroneamente ou tentavam efetuar uma jogada somente utilizando peças com quantidades e configurações idênticas aquelas das extremidades do jogo. Isso também serve para explicar o paradoxo relativo à menor prevalência do erro E7 em G1 (PTBP) do que em G2 (AT). Ora, se G1 (PT-BP) contava quase todas as suas peças visando a escolher a "ideal", as chances de não incorrerem nesse erro tornavam-se muito maiores.

\section{REFERÊNCIAS}

1. Organização Mundial de Saúde - OMS [homepage]. CID-10: Classificação internacional das doenças. [acesso em 25 mar 2008]. Disponível em http:// www.datasus.gov.br/cid10/webhelp/ cid10.htm

2. Abreu LC, Souza AMB, Oliveira AG et al. Incidência de hemorragia periintraventricular em recém nascidos prétermo e a relação com o peso ao nascer. Rev. bras. crescimento desenvolv. hum., 2007; vol.17(2): 24-30.

3. Oliveira AG, Siqueira PP, Abreu LC. Cuidados nutricionais no recém-nascido de muito baixo peso. Rev. bras. crescimento desenvolv. hum., 2008; vol.18(2): 148-154.
Conclui-se então que, se os objetivos desta pesquisa foram os de verificar se crianças nascidas prematuras e com baixo peso, em idade pré-escolar, apresentam noção de conservação de quantidades discretas e se prematuridade e baixo peso ao nascer estão relacionados com dificuldades na construção da noção de conservação de quantidades discretas e formação do conceito de número, pode-se afirmar que: a) os resultados e análises não permitem demonstrar que a noção de conservação de quantidades discretas está consolidada nos participantes, tanto de G1 (PT-BP) como de G2 (AT); b) a pesquisa não encontrou diferenças estatisticamente significativas que demonstrem correlações entre prematuridade e baixo peso ao nascer com dificuldades na construção da noção de conservação de quantidades discretas e formação do conceito de número, posto que os níveis de operacionalidade apresentados pelos dois grupos avaliados foram semelhantes, demonstrando incompletude da referida noção.

4. Ministério da Saúde - MS [homepage]. Estatísticas vitais - mortalidade e nascidos vivos. [acesso em 20 fev 2008]. Disponível em http://tabnet.datasus.gov.br/ cgi/tabcgi.exe?sinasc/cnv/nvuf.def

5. Magalhães LC, Catarina PW, Barbosa VM, Mancini MC, Paixão ML. Estudo comparativo sobre o desempenho perceptual e motor na idade escolar em crianças nascidas pré-termo e a termo. Arq Neuro. 2003; 61(2-A): 250-255.

6. Bhutta AT, Cleves MA, Casey PH, Cradock MM, Anand KJS. Cognitive and behavioral outcomes of school-aged children who were born preterm. JAMA. 2002; 288(6): 728-737.

7. Lubchenco LO. Determinação do peso e idade gestacional. In Avery GB, organizador. Neonatologia. Rio de Janeiro: Medsi, 1984. p. 207-227. 
8. Chaudhari S, Kulkarni S, Pajnigar F, Pandit AN, Descmukh SA. Longitudi-nal follow-up of development of preterm infants. J Indian Acad Pediatrics. 1991; 28(8): 873-880.

9. D’Angio CT, Sinkin RA, Stevens TP, Landfish NK, Merzbach JL, Ryan RM, et al. Longitudinal, 15-year follow-up of children born at less than 29 weeks' gestation after introduction of surfactant therapy into a region: neurologic, cognitive, and educational outcomes. Pediatrics. 2002; 110: 1094-1102.

10. Martinez CMS, Joaquim, RHVT, Oliveira EB, Santos IC. Suporte informacional como elemento para orientação de pais de pré-termo: um guia para o serviço de acompanhamento do desenvolvimento no primeiro ano de vida. Rev Bras Fisioter. 2007; 11(1): 73-81.

11. Linhares MBM, Carvalho AEV, Bordin MBM, Chimello JT, Martinez FE, Jorge SM. Prematuridade e muito baixo peso como fatores de risco ao desenvolvimento da criança. Cad Psicol e Saude Paideia. 2000; 10(18): 60-69.

12. Damman O, Walther H, Allers B, Schroder M, Drescher J, Lutz D, et al. Development of a regional cohort of very-lowbirthweight children at six years: Cognitive abilities are associated with neurological disability and social background. Dev Med Child Neurol. 1996; 38: 97-108.

13. Nadeau L, Boivin M, Tessier R, Lefebvre F, Robaey P. Mediators of behavioral problems in 7 year old children born after 24 to 28 weeks of gestation. J Dev Behav Pediatr. 2001; 22(1): 1-10.

14. Rodrigues MCC, Mello RR, Fonseca SC. Dificuldade de aprendizagem em escolares de muito baixo-peso ao nascer. J Pediat. 2006; 82(1): 6-14.

15. Taylor HG, Klein N, Minhch NM, Hack M. Middle-school-age outcomes in children with very low birthweight. Child Dev. 2000; 71: 1495-1511.
16. Kramer MS. Determinants of low birth weight: methodological assessment and meta-analysis. Bull World Health Org. 1987; 65: 663-737.

17. Klein VC, Linhares MBM. Prematuridade e interação mãe-criança: revisão sistemática da literatura. Psicol Estud. 2006; 11(2): 277-284.

18. Linhares MBM, Chimello JT, Bordin MBM, Carvalho AEV, Martinez FE. Desenvolvimento psicológico na fase escolar de crianças nascidas pré-termo em comparação com crianças nascidas a termo. Psicol: Reflexao e Critica. 2005; 18(1): 109-117.

19. Reichman NE. Low birth weight and school readiness. Fut Child. 2005; 15: 91116.

20. Rodrigues MCC, Mello RR, Fonseca SC. Dificuldade de aprendizagem em escolares de muito baixo-peso ao nascer. J Pediatr. 2006; 82(1): 6-14.

21. Bismarck-Nasr EM, Frutuoso MFP, Gamabardella AMD. Efeitos tardios do baixo peso ao nascer. Rev Bras Crescimento Desenvolv Hum. 2008; 18(1): 98-103.

22. Anderson P, Doyle LW. Neurobehavioral outcomes of school-age children born extremely low birth weight or very preterm in the 1990s. JAMA. 2003; 289(24): 32643272.

23 Rugolo LMSS. Crescimento e desenvolvimento a longo prazo do prematuro extremo. J Pediatr. 2005; 81 Supl 1: 101-110.

24. Kamii C. A criança e o número: implicações educacionais da teoria de Piaget para a atuação com escolares de 4 a 6 anos. 32a ed. Campinas: Papirus, 2005.

25. Kamii C. Crianças pequenas continuam reinventando a Aritmética: Implicações da teoria de Piaget. 2a ed. Campinas: Papirus, 2005.

26. Kamii C, Livingston SJ. Desvendando a Aritmética: implicações da teoria de Piaget. 6a ed. Campinas: Papirus, 2001. 
27. Piaget J. Seis estudos de Psicologia. 9a ed. Rio de Janeiro: Forense Universitária; 1978.

28. Monteiro G, Medeiros JG. A contagem oral como pré-requisito para a aquisição do conceito de número com crianças pré-escolares. Estud Psico. 2002; 7(1): 73-90.

29. Piaget J, Szeminska A A gênese do número na criança. Rio de Janeiro: Zahar, 1975.

30. Assis MBC. Procedimentos de escolha conforme o modelo e escolha do ímpar na aquisição da noção de conservação [dissertação]. São Paulo (SP): Universidade de São Paulo; 1981.

31. Piaget J. O possível e o necessário: a evolução dos necessários na criança. Porto Alegre: Artmed, 1986.

32. Macedo L. Ensaios construtivistas. São Paulo: Casa do Psicólogo, 1994.
33. Castorina JA. Psicologia genética: aspectos metodológicos e implicações psicopedagógicas. Porto Alegre: Artmed, 1988.

34. Delval J. Introdução à prática do método clínico: descobrindo o pensamento das crianças. Porto Alegre: Artmed, 2002.

35. Piaget J. A representação do mundo na criança. São Paulo: Idéias e Letras, 2005.

36. Queiroz SS. Inteligência e afetividade na dialética de Jean Piaget: um estudo com o jogo da senha [tese]. São Paulo (SP): Universidade de São Paulo; 2000.

37. SPSS. Statistical package for social sciences for windows [software]. Base 15.0. Chicago (IL): SPSS; 2006.

Recebido em 01 de setembro de 2009 Modificado em 26 de setembro de 2009 Aceito em 14 de novembro de 2009 